\title{
Modelling Turbulence Effect in Formation of Zonal Winds
}

\author{
J. Heinloo and A. Toompuu*
}

Marine Systems Institute, Tallinn University of Technology, Akadeemia tee 21, 12618 Tallinn, Estonia

\begin{abstract}
A turbulence-affected mechanism of formation of zonal winds in the Earth's troposphere is discussed from the perspective of the theory of rotationally anisotropic turbulence (the RAT theory). The turbulence effect is explained as an action of the turbulence rotational viscosity introduced within the RAT theory to characterize the shear in relative rotation (determined as the difference between the average angular velocity of eddy rotation and the vorticity of the average velocity field). The effect manifests in the form of an additive correction to the wind velocity predicted by the geostrophic approach. It is shown that the accounted turbulence effect decreases the westerlies' velocity predicted by the geostrophic approach at lower latitudes and can be used to explain the formation of easterlies (trade winds) in the equatorial zone without any necessity of assigning the geopotential a local minimum, at the Equator which is required to explain the trade winds within the purely geostrophic approach.
\end{abstract}

Keywords: Turbulence, general circulation, climatology.

\section{INTRODUCTION}

Predominantly zonal winds (ZW) in the Earth's atmosphere (westerlies at middle latitudes and trade winds in the equatorial zone) are of special interest not only because of their global significance to the Earth's climate and navigation, but also because they represent a motion pattern common to all planetary atmospheres. One of the problems of $\mathrm{ZW}$ requiring additional discussion is the turbulence effect in the formation of $\mathrm{ZW}$. Although the contemporary hydromechanics have developed several methods for such discussion, most of these methods are founded on capacious numerical procedures, presentings the situation with great number of details accounted [1-4] which may mask the physical essence of the effects under investigation. In order to present more distinctly the turbulence effect in $\mathrm{ZW}$ formation it is appropriate to complement numerical methods with a description set up immediately in terms of average quantities. Nevertheless, the conventional turbulence mechanics (CTM), supposed to be found this type of turbulence descriptions, does not meet expectations. In particular, CTM does not account for effects caused by the preferred orientation of eddy rotation, which remarkably constricts its applicability. The theory in [5-9] (concisely outlined in Section 2 as the rotationally anisotropic turbulence (RAT) theory) removes the constriction. The RAT theory treats the turbulence according to the Richardson-Kolmogorov turbulence conception [21, 22] as a stochastic form of fluids motion constituted by a hierarchy of eddies of different scales, whereas the eddies of the largest scales, immediately interacting with the average flow, have a preferred orientation of rotation.

By accounting for effects of the preferred orientation of eddy rotation the RAT theory augments the turbulence average description and provides the turbulent media with

*Address correspondence to this author at the Marine Systems Institute, Tallinn University of Technology, Akadeemia tee 21, 12618 Tallinn, Estonia; Tel: 372 6204306; Fax: +372 6204301; E-mail: heinloo@phys.sea.ee additional physical properties. The current paper exploits one of these properties, the turbulence rotational viscosity, for outlining the turbulence effect in formation of the $\mathrm{ZW}$ velocity field in the Earth's troposphere. The discussion is performed within a model similar to the model $[10,11]$ treating the turbulence effect in the formation of the Antarctic Circumpolar Current velocity field. This paper discusses the turbulence effect in the ZW formation in the Earth's atmosphere which complements former geophysical applications of the RAT theory. In addition to the mentioned Antarctic Circumpolar Current problem, the problems of the ocean upper layer dynamics $[12,13]$, of the formation of the Gibraltar Salinity Anomaly [14], of the formation of flows over varying bottom topography [15] and of the momentum transfer and energy conversion processes in the Gulf Stream area [16] have been considered.

\section{The RAT Theory}

The RAT theory [5-9] realizes a generalized setup of turbulence average description proceeding from distinguishing of the velocity fluctuations at each flow field point (in addition to their magnitude and orientation) by the curvature of the velocity fluctuation streamlines passing the point. The generalization is formalized by inclusion of the curvature radius of the velocity fluctuation streamlines $\mathbf{R}$ into the set of arguments of the probability distribution, specifying the applied averaging. $\quad\left(\boldsymbol{R}=\partial \boldsymbol{e} / \partial s|\partial \boldsymbol{e} / \partial s|^{-2}, \quad\right.$ where $\boldsymbol{e}=\boldsymbol{v}^{\prime} / v^{\prime}$, in which $\boldsymbol{v}^{\prime}$ is the velocity fluctuation, $v^{\prime}=\left|\boldsymbol{v}^{\prime}\right|$, and $s$ is the length of the $\boldsymbol{v}^{\prime}$ streamline curve).

The specified averaging permits the definition of quantity $\boldsymbol{M}=\langle\boldsymbol{v} \times \boldsymbol{R}\rangle$ (angular brackets denote statistical averaging in the sense specified above) having the physical sense of internal moment of momentum (angular momentum, spin) of the turbulent flow field and playing the role of a dynamical 
measure of a preferred orientation of eddy rotation. The definition of $\boldsymbol{M}$ is coupled with definition

$\boldsymbol{\Omega}=\left\langle\boldsymbol{v}^{\prime} \times \frac{\boldsymbol{R}}{\boldsymbol{R}^{2}}\right\rangle=\left\langle\boldsymbol{v}^{\prime} \times \frac{\partial \boldsymbol{e}}{\partial \mathrm{s}}\right\rangle$.

Quantity $\boldsymbol{\Omega}$ has the sense of the average angular velocity of eddies' rotation. It is natural to interconnect the moment of momentum $\boldsymbol{M}$ with the angular velocity of rotation $\boldsymbol{\Omega}$ by relation $\boldsymbol{M}=J \boldsymbol{\Omega}$, where $\boldsymbol{J}$ defines the effective moment of inertia, the square root of which determines the characteristic spatial scale of eddies contributing to the preferred orientation of eddy rotation.

The description of motion of turbulent media with $\boldsymbol{M} \neq 0$ should be set up within the conservation laws for the average momentum (the Reynolds equation), for the moment of momentum $\boldsymbol{M}$ and for energy $K^{0}$, determined as the difference between the total turbulent energy $K=\frac{1}{2}\left\langle v^{\prime 2}\right\rangle$ and energy $K^{\Omega}=\frac{1}{2} \boldsymbol{M} \cdot \boldsymbol{\Omega}$. The turbulent stress tensor is determined within the suggested setup of turbulence mechanics as asymmetric with the antisymmetric constituent describing the interaction of the average flow with the turbulence constituent contributing to the condition $\boldsymbol{M} \neq 0$. The asymmetry of the turbulent stress tensor declares that the condition $\boldsymbol{M} \neq 0$ turns (in general) the probability distribution non-invariant in respect to permutation of its arguments - the velocity fluctuation components.

The closure problem, immanent to any average turbulence description, is solved in the RAT theory in agreement with the methodology applied in formulation of descriptions of micropolar fluids [17-20]. The methodology connects the generalized forces of the description linearly with the corresponding generalized velocities. In particular, the applied method connects the dual vector to the antisymmetric constituent of the turbulent stress tensor $\boldsymbol{\sigma}$ (the components of $\boldsymbol{\sigma}$ are defined as $\sigma_{k}=e_{i j k} R_{i j}$, where $e_{i j k}$ denote the components of Levi-Civita tensor, $R_{i j}$ denote components of the turbulent stress tensor and the Einstein summation is assumed) to the angular velocity of relative rotation $\boldsymbol{\Omega}-\boldsymbol{\omega}$, where $\boldsymbol{\omega}=\frac{1}{2} \nabla \times \boldsymbol{u}$ is the vorticity of the average flow ( $\boldsymbol{u}=\langle\boldsymbol{v}\rangle$, where $\boldsymbol{v}$ is actual velocity and the coefficient $1 / 2$ in the definition of vorticity is included for terminological convenience), by

$\sigma=4 \gamma(\Omega-\omega)$.

Coefficient $\gamma>0$ (characterizing the shear in relative rotation) introduced in (1) is the coefficient of rotational viscosity having the dimension of kinematic velocity $\left(\mathrm{m}^{2} \mathrm{~s}^{-1}\right)$ but differing from the latter by its physical sense.

The quoted in the introduction geophysical models have applied the RAT theory within a simplification follow- ing from the Richardson-Kolmogorov turbulence conception $[21,22]$. The simplification stands in neglecting the turbulence constituent not contributing to the preferred orientation of eddy rotation in the average flow-turbulence interaction description. In terms of the turbulence viscosity it means considering the effects of the turbulence shear viscosity, which mediates the average flow interaction with the turbulence constituent not contributing to the preferred orientation of eddy rotation, negligibly small if compared with the effects of the turbulence rotational viscosity, which realizes the average flow interaction with the turbulence constituent contributing to the preferred orientation of eddy rotation. The present paper applies the same simplification.

\section{MODEL OF ZONAL WINDS IN THE EARTH'S ATMOSPHERE}

\subsection{Model Setup}

The model specifies the Earth's atmosphere as a turbulent gas layer covering the Earth (modeled as a solid sphere rotating with angular velocity $\boldsymbol{\omega}^{0}$ ) with the thickness much smaller than the Earth's radius $r$. The motion is considered in the right-hand spherical co-ordinate system $(\varphi, \vartheta, z)$, where $\varphi$ is longitude, $\vartheta$ is latitude $\left(-\frac{\pi}{2}<\vartheta<\frac{\pi}{2}\right)$ and $z$ is height (with $z=0$ on the solid sphere). It is assumed that density $\rho$ and the wind velocity $\boldsymbol{u}$, determined as statistically average quantities, are specified as $\rho=\rho(\vartheta, z)$ and $\boldsymbol{u}=(u(\vartheta, z), 0,0)$, i.e. do not depend on the zonal direction.

The suggested model is aimed to describe the effect of a preferred orientation of turbulent eddies rotation on the zonal winds formation. As including notions introduced within the RAT theory the model treats the RAT theory (Section 2) as an inseparable part of the discussion.

\subsection{Geostrophic Model of ZW}

Firstly consider stationary balance condition

$-\nabla p+2 \rho \boldsymbol{u} \times \boldsymbol{\omega}^{0}+\rho \boldsymbol{g}=0$,

neglecting the turbulence viscosity effects. In (2), besides the quantities explained above, $\nabla=\left(r^{-1}(\cos \vartheta)^{-1} \partial / \partial \varphi, r^{-1} \partial / \partial \vartheta, \partial / \partial z\right)$, $p$ is the average pressure and $g=(0,0,-g)$ is the gravitational acceleration. The geostrophic model of ZW stems from (2) for the vertical constituent of the Coriolis force considered as negligibly small with respect to $\rho g$ and $p_{, z}$. From (2) in this case it follows that $p_{, z}=-\rho g$ and $u=-p_{, \vartheta} / \rho r f$, where $f=2 \omega^{0} \sin \vartheta$ is the Coriolis parameter and the index following comma denotes differentiation with respect to the corresponding coordinate. Using geopotential $\Phi=g z$, connected with the pressure by $\partial \Phi / \partial \vartheta=\rho^{-1} \partial p / \partial \vartheta$, the expression for the wind velocity $u$ can be written as 
$u=-\frac{\Phi_{, \vartheta}}{r f}$,

Differentiating (3) in respect to $\vartheta$ and accounting for $\sin \vartheta=0$ and $\cos \vartheta=1$ at the Equator we have from (3)

$u(0)=-\left.\frac{1}{2 \omega^{0}} \Phi_{, \vartheta \vartheta}\right|_{\vartheta=0}$.

Expressions (3) and (4) show that the wind velocity $u$, predicted by the geostrophic approach, can agree with the observed easterlies in the equatorial region only if the geopotential extremum, specified as the local minimum, is located at the Equator.

In order to estimate the effect of restrictions imposed to the geopotential behavior by the geostrophic approach let us consider the geopotential distribution (Fig. 1) calculated by formula

$\Phi=-R \int_{p_{0}}^{p} T d \ln p$

from [23], where $R=287 \mathrm{~J} \mathrm{~kg}^{-1} \mathrm{~K}^{-1}$ is the gas constant,

$T$ is the zonal average air temperature (measured in $\mathrm{K}$ ) and $p_{0}$ is the pressure at $z=0$, from the data set from the period 1958-1973 [24]. The estimated geopotential $\Phi(\vartheta)$ (Fig. 1) and the calculated profiles of its first longitudinal derivative, $\Phi_{\vartheta \vartheta}$ for $-15^{\circ}<\vartheta<15^{\circ}$, depicted in Fig. (2), show that the geopotential extremum appears to be maximum and is located to the north of Equator i.e. the geostrophic approach to the $\mathrm{ZW}$ calculation does not comply with the geopotential distribution estimated from data.

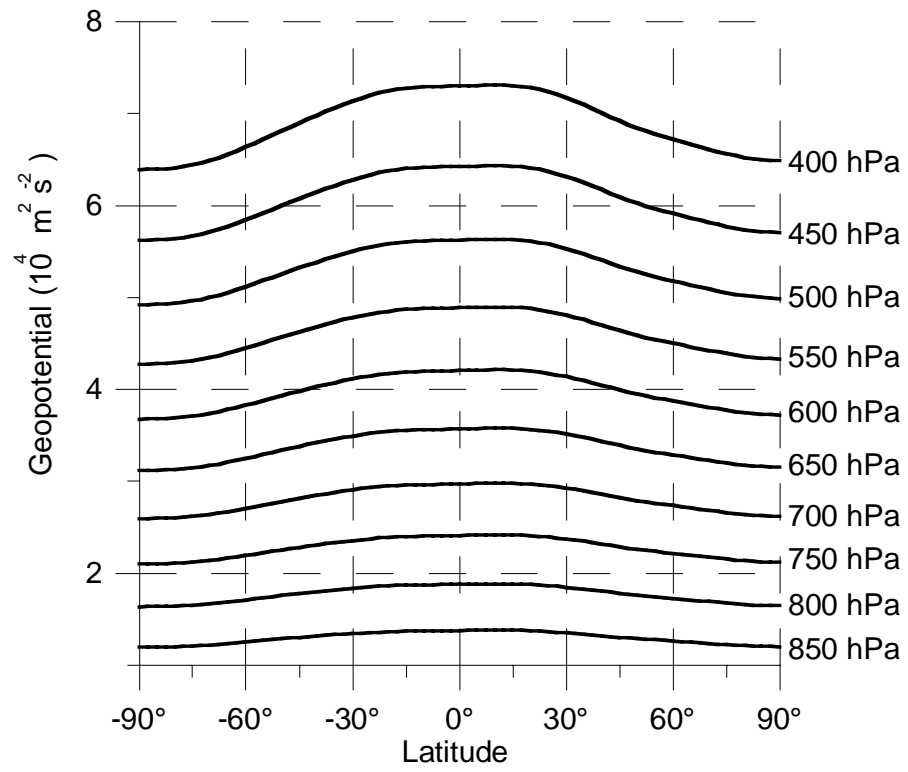

Fig. (1). Latitudinal distribution of zonal mean geopotential $\Phi(\vartheta)$ for pressure levels 400 to $850 \mathrm{hPa}$ estimated from data compiled by Oort (1983).

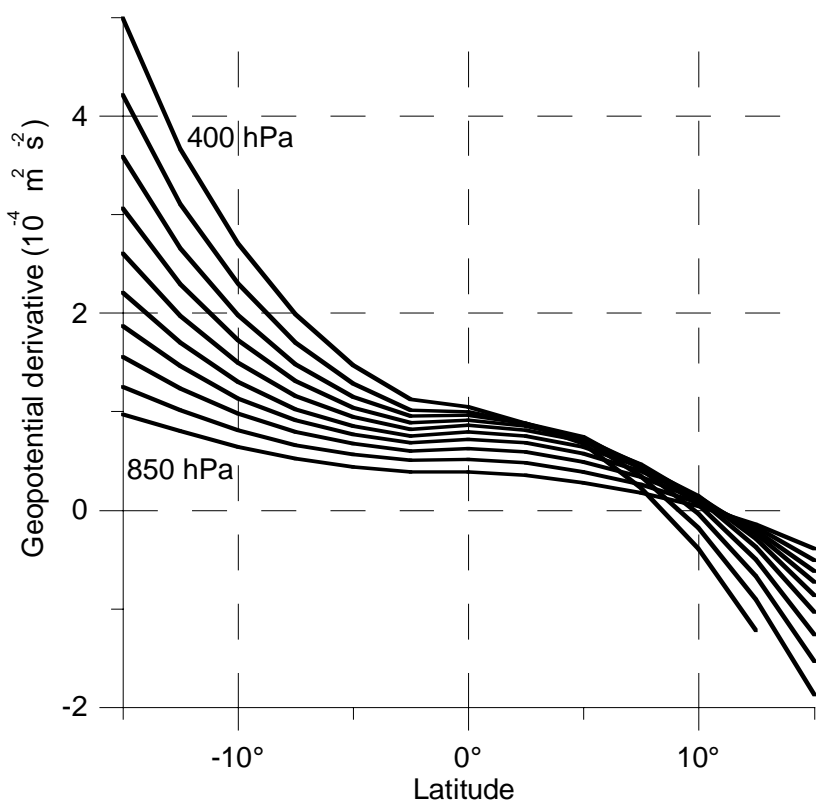

Fig. (2). Zonal geopotential derivative in respect to latitude, $\Phi(\vartheta)_{, \vartheta}$ for pressure levels 400 to $850 \mathrm{hPa}$ with step $50 \mathrm{hPa}$.

In order to harmonize the geopotential distribution with the observed meridional velocity distribution in the geostrophic approach it has been rather common so far to accept a relatively small (in the limits of assumed estimation error) modification of geopotential values in the near-Equatorial region to satisfy the required criteria (see the COSPAR International Reference Atmosphere (CIRA-86) reanalysis [25] for troposphere based on data compiled by Oort [24] and the reanalysis data from 1968-1996 provided by the NOAACIRES Climate Diagnostics Center, Boulder, Colorado, on their web site at http://www.cdc.noaa.gov).

\subsection{Turbulence Effect in the ZW Formation}

It is commonly believed [23] that accounting for the turbulence effect can overcome the shortcomings of the geostrophical model. In this case the balance condition (2) is replaced by

$-\nabla p+\boldsymbol{F}+2 \rho \boldsymbol{u} \times \boldsymbol{\omega}^{0}+\rho \boldsymbol{g}=0$,

where $\boldsymbol{F}$ is the divergence of the turbulent stress tensor, the components $F_{i}$ of which are defined as $F_{i}=R_{i j, j}$, where the index following comma denote differentiation in respect to the corresponding spatial coordinate and the Einstein's summation is assumed. The balance condition (6) requiring a specification of $\boldsymbol{F}$ relates the $\mathrm{ZW}$ description to the specifics of the selected turbulence model. Therefore, dependent on the selected turbulence model, the balance condition (6) can be used to found different particular $\mathrm{ZW}$ models accounting for the turbulence effect.

In the model discussed in the following, $\boldsymbol{F}$ is specified according to the RAT theory as

$\boldsymbol{F}=\frac{1}{2} \nabla \times \boldsymbol{\sigma}$ 
where $\boldsymbol{\sigma}$ is determined in (1) $\left(F_{i}=R_{i j, j}=\frac{1}{2} e_{i j k} \sigma_{k, j}\right)$. Substituting $\boldsymbol{F}$ from (7) into (6) we have

$-\nabla p+\frac{1}{2} \nabla \times \boldsymbol{\sigma}+2 \rho \boldsymbol{u} \times \boldsymbol{\omega}^{0}+\rho \boldsymbol{g}=0$,

Equation (8) is similar to the respective equation exploited [11] in the context of discussion of the turbulence effect in the formation of the Antarctic Circumpolar Current velocity field. Unlike the discussion in [11] equation (8) is not restricted by the Boussinesq approximation.

Let $\boldsymbol{u}$ in (8) be presented as the sum

$\boldsymbol{u}=\boldsymbol{u}^{\mathrm{g}}+\boldsymbol{u}^{\mathrm{ag}}$,

where $\boldsymbol{u}^{\mathrm{g}}=\left(u^{g}, 0,0\right)$ is the geostrophic velocity constituent satisfying (8) for the turbulence effect absent and $\boldsymbol{u}^{\mathrm{ag}}=\left(u^{a g}, 0,0\right)$ is the ageostrophic turbulence-induced correction to $\boldsymbol{u}^{\mathrm{g}}$. Accounting for (1) with $\omega_{\varphi}=0$ and assuming that the medium turbulence does not violate the hydrostatic balance condition (3), from the vertical projection of (8) we have

$u^{a g}=\frac{\gamma}{\rho r \omega^{0} \cos ^{2} \vartheta}\left(\Omega_{\varphi} \cos \vartheta\right)_{, \vartheta}$.

Expression (9) specifies the turbulence effect on the zonal wind velocity as caused by the turbulence rotational viscosity $(\gamma \neq 0)$. (For $u^{\mathrm{ag}}$ from (9) the meridional projection of (8) determines the turbulence-caused meridional pressure force constituent while the zonal projection of (8) establishes the relation between $\sigma_{z}$ and $\sigma_{\vartheta}$.) Let us stress that unlike discussed in 3.2. geostrophic approach neglecting the vertical constituent of the Coriolis force, the deduction of expression (9) just makes use of this Coriolis force vertical constituent.

To determine $\Omega_{\varphi}$ equation (8) should be complemented by the equation for the moment of momentum $\boldsymbol{M}$. Neglecting, similar to the model discussed in [11], the effects of diffusion of the moment of momentum $\boldsymbol{M}$, for the body force acting on the medium specified as the gravitational force, this equation in a non-rotating frame reads as [5-7]

$\rho \frac{d}{d t} \boldsymbol{M}=-\boldsymbol{\sigma}-4 \kappa \boldsymbol{\Omega}+\rho \boldsymbol{m}+\rho J(\nabla \boldsymbol{u}) \cdot \boldsymbol{\Omega}$,

where $d / d t=\partial / \partial t+\boldsymbol{u} \cdot \nabla, \kappa$ is the coefficient measuring the effect of $\boldsymbol{M}$ decay due to the cascade scattering and

$\rho m=g \times\left\langle\rho^{\prime} \boldsymbol{R}\right\rangle$.

When written in the coordinate system rotating with angular velocity $\boldsymbol{\omega}^{0}$ the quantities $\boldsymbol{\Omega}, d \boldsymbol{M} / d t$ and $\rho J(\nabla \boldsymbol{u}) \cdot \boldsymbol{\Omega} \quad$ in $\quad(10)$ are replaced by $\boldsymbol{\Omega}+\boldsymbol{\omega}^{0}$, $d \boldsymbol{M} / d t+\omega^{0} \times \boldsymbol{M}$ and $\rho J(\nabla \boldsymbol{u}) \cdot\left(\boldsymbol{\Omega}+\boldsymbol{\omega}^{0}\right)+\rho J \boldsymbol{\Omega} \times \boldsymbol{\omega}^{0}$ respectively and for the steady and zonally homogenous $\boldsymbol{M}$ from (9) it follows

$$
\begin{aligned}
& -\sigma-4 \kappa\left(\Omega+\omega^{0}\right)+\rho m+\rho J(\nabla u) \cdot\left(\Omega+\omega^{0}\right) \\
& +2 \rho J \Omega \times \omega^{0}=0
\end{aligned}
$$

from which, for the last two terms on the left side of (12) considered to be small if compared to the other terms of (12), we shall have

$$
-\sigma-4 \kappa\left(\Omega+\omega^{0}\right)+\rho m=0 .
$$

(The balance condition (13) differs from the corresponding balance condition applied in [11] only by specification of the density which is not restricted in (13) by the Boussinesq approximation.) The specification of $\left\langle\rho^{\prime} \boldsymbol{R}\right\rangle$ in the form [9, 11]

$\left\langle\rho^{\prime} \boldsymbol{R}\right\rangle=k_{1} \nabla \rho \times \boldsymbol{\Omega}-k_{2} \nabla \rho$

determines $\rho \boldsymbol{m}$, specified by (11), in (13) as

$$
\left.\rho \boldsymbol{m}=-k_{1}[\nabla \rho \cdot \boldsymbol{g}) \hat{\boldsymbol{I}}-(\nabla \rho) \boldsymbol{g}\right] \cdot \boldsymbol{\Omega}+k_{2} \nabla \rho \times \boldsymbol{g},
$$

which closes (13) to determine $\boldsymbol{\Omega}$. In (15) $\hat{\boldsymbol{I}}$ is unit tensor. From (15) it follows that coefficients $k_{1}$ and $k_{2}$, introduced by (14), quantify the effects of stratification and baroclinic instability on the turbulent eddy rotation, respectively. As far as the stable stratification suppresses and the baroclinic instability feeds the turbulence, the coefficients $k_{1}$ and $k_{2}$ are determined positive.

Using (15) and (1) from (13) we shall have

$$
\begin{aligned}
& \Omega_{\varphi}=-\frac{c_{2}}{r} \frac{\rho_{, \vartheta}}{1-c_{1} \rho_{, z}} ; \\
& \Omega_{\vartheta}=\frac{1}{\gamma+\kappa} \frac{\gamma \omega_{\vartheta}-\kappa \omega^{0} \cos \vartheta}{1-c_{1} \rho_{, z}}+\frac{c_{1}}{c_{2}} \Omega_{z} \Omega_{\varphi} ; \\
& \Omega_{z}=\frac{1}{\gamma+\kappa}\left(\gamma \omega_{z}-\kappa \omega^{0} \sin \vartheta\right),
\end{aligned}
$$

where $c_{1}=k_{1} g / 4(\gamma+\kappa), \quad c_{2}=k_{2} g / 4(\gamma+\kappa)$. Accounting for (16), expression (9) for $u^{\text {ag }}$ can be rewritten as

$$
u^{a g}=-\frac{c_{2} \gamma}{\rho r^{2} \omega^{0} \cos ^{2} \vartheta}\left(\frac{\rho_{, \vartheta} \cos \vartheta}{1-c_{1} \rho_{, z}}\right)_{, \vartheta} .
$$

Assuming the scaling property of turbulence, the coefficients $\gamma, \kappa, k_{1}$ and $k_{2}$ appear proportional to the effective moment of inertia $J$ [8] and therefore the expressions (16) (19) depend on the spatial scale $\ell=\sqrt{J}$ of the turbulence constituent contributing to the preferred orientation of eddy 
rotation. In particular, from (19) it follows that $u^{a g}$ is proportional to $J=\ell^{2}$.

\subsection{Model Analysis}

Consider a situation with horizontally homogeneous density field and with the stratification strong enough to depress $\Omega_{\varphi}$ and $\Omega_{\vartheta}$ i.e. stating that $\Omega_{\varphi}=\Omega_{\vartheta}=0$. According to (9) and (18) we have in this case $u^{\mathrm{ag}}=0$ and $\Omega_{z} \neq 0$. The latter condition means the turbulence with the average angular velocity of eddy rotation orientated vertically. The situation changes if the density varies in meridional direction with the meridional gradient large enough to generate $\Omega_{\varphi}$. Consider $\Omega_{\vartheta}$ still remaining suppressed by the stratification. It is easy to imagine the generation of $\Omega_{\varphi}$, and consequently of $u^{\mathrm{ag}}$, in this case as accompanied by an inclination of the preferred orientation of turbulent eddies' axes from their vertical direction, caused by the nonhomogenous meridional density distribution.

To analyze the structure of $u^{\mathrm{ag}}$ predicted by (19) consider pressure $p$ and density $\rho$ exponentially decreasing with height $p=p_{0} \exp (-\alpha z)$ and $\rho=\rho_{0}(\vartheta) \exp (-\alpha z)$, where $p_{0}$ and $\rho_{0}(\vartheta)$ denote pressure and density on the reference level $(z=0)$, while $\rho_{0}(\vartheta)$ is symmetrical in respect to the equator and increases to the poles with its gradient vanishing at the equator and at the poles. In terms of variables $\vartheta$ and $p$ we have for $\rho$ in this case

$\rho=\rho(\vartheta, p)=\rho_{0}(\vartheta) \frac{p}{p_{0}}$

where $\rho_{0}(\vartheta)=\rho\left(p=p_{0}, \vartheta\right)$. Expression (20) determines the meridional density distributions at different pressure levels as self-similar. It reduces the density distributions on different pressure levels to a single density distribution on the reference level. Using (20) and (3) for $\rho_{, z}$ in (19) we shall have

$\rho_{, z}=\frac{\partial \rho}{\partial p} p_{, z}=-g \frac{\rho_{0}(\vartheta)}{p_{0}} \rho=-\frac{\beta}{c_{1}}\left(\rho_{0}(\vartheta)\right)^{2} \frac{p}{p_{0}}$

where $\beta=c_{1} g / p_{0}=$ const and expression (19) for $u^{\mathrm{ag}}$ shall become

$u^{\mathrm{ag}}=\frac{\gamma c_{2}}{r^{2} \omega^{0}} f(\vartheta, p)\left[\beta f(\vartheta, p) \frac{p}{p_{0}} F_{1}(\vartheta)-F_{2}(\vartheta)\right]$

in which

$$
F_{1}(\vartheta)=\frac{2}{\cos \vartheta}\left(\rho_{0}(\vartheta)_{, \vartheta}\right)^{2}>0
$$

and

$$
F_{2}(\vartheta)=\frac{1}{\rho_{0}(\vartheta) \cos ^{2} \vartheta}\left(\rho_{0}(\vartheta)_{, \vartheta} \cos \vartheta\right)_{, \vartheta}
$$

are functions determined by the density distribution at the reference level, and

$$
f(\vartheta, p)=\frac{1}{1+\beta\left(\rho_{0}(\vartheta)\right)^{2} \frac{p}{p_{0}}}
$$

For the constituted above properties of $\rho_{0}(\vartheta)$, function $F_{1}(\vartheta)$ appears positive with two maximums located at inflection points of the density meridional distribution and with zero value at the equator. Unlike $F_{1}(\vartheta)$, function $F_{2}(\vartheta)$ is zero at the inflection points and has three extremums - at the equator and one on each side of the equator between the maximums of $F_{1}(\vartheta)$ and the poles. For the density increasing to the poles $F_{2}(\vartheta)$ is negative at the equator. Expression (21) specifies $u^{\mathrm{ag}}$ as a linear combination of $F_{1}(\vartheta)$ and $F_{2}(\vartheta)$ with their relative weights determined by $f(\vartheta, p)$.

The sketched structure of $u^{\mathrm{ag}}$ displays the turbulence effect on the ZW as increasing the wind velocity at higher latitudes and decreasing the wind velocity at lower latitudes. The decrease includes the formation of negative $u^{\mathrm{ag}}$ in the equatorial zone i.e. the turbulence can explain the equatorial easterlies. The increase of the wind velocity at higher latitudes and its decrease at lower latitudes is accompanied with a shift of the location of the westerlies maximums to the poles if compared with the prediction of purely geostrophic approach. Let us note that although $\gamma \propto \ell^{2}$ depends on $\vartheta$ (due to the presumed dependence of $\ell$ on $\vartheta$ ), this dependence does not change the assertions made.

\subsection{The Model Applied to the Actual Atmospheric Data}

Fig. (3) collects the data for $\rho_{0}(\vartheta)$ calculated from

$\rho(\vartheta, p)=\rho_{0}(\vartheta) \frac{p}{p_{0}}+\rho^{*}$

where $\rho^{*}=0.127 \mathrm{~kg} \mathrm{~m}^{-3}, \quad p_{0}=1000 \mathrm{hPa} \quad$ and $\rho(\vartheta, p)$ is the average density meridional distribution estimated from the temperature and humidity data compiled by Oort [24] for the pressure levels from 400 to $850 \mathrm{hPa}$ with the step of $50 \mathrm{hPa}$. The data are approximated by $\rho_{0}(\vartheta)=a_{0}-a_{1} \exp \left(-b_{1} \vartheta^{4}\right)-a_{2} \exp \left(-b_{2} \vartheta^{2}\right), \quad$ where 


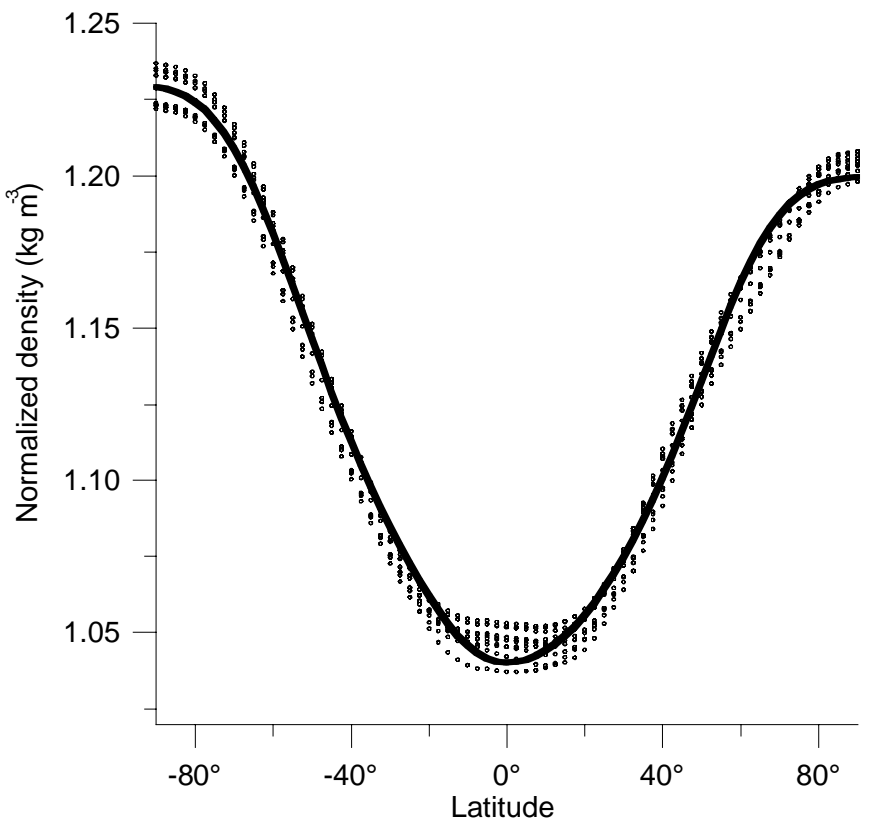

Fig. (3). Normalized zonal density $\rho_{0}(\vartheta)$ vs. latitude (dots) and its approximation (curve).

$a_{0}, a_{1}, b_{1}, a_{2}, b_{2}$ are constants (curve in Fig. (3)). Fig. (4) presents calculations for $u^{g}$ (dashed curves) and $u=u^{\mathrm{g}}+u^{\mathrm{ag}}$ (curves) together with the wind data (markers) for 400,600 and $850 \mathrm{hPa}$. The calculations are performed for the northern hemisphere, which is much more densely populated by the original observation data than the southern hemisphere $[24,26]$. The geostrophic wind is calculated from the geopotential shown in Fig. (1) modified in the interval $-15^{\circ}<\vartheta<15^{\circ}$ to make the geopotential maximum location coinciding with the Equator. The geopotential values alteration within the indicated latitude interval did not exceed $0.3 \%$. The ageostrophic wind constituent $u^{\mathrm{ag}}$ is calculated from (19) with (22) by using the approximated $\rho_{0}(\vartheta)$ for the meridional dependence of $\ell$ on $\vartheta$ modeled as $\ell=\ell_{0} \cos \vartheta$, for $\beta=c_{1} g / p_{0}=1 \mathrm{~m}^{6} \mathrm{~kg}^{-2}$ and $\frac{c_{2} \gamma}{r^{2} \omega^{0}}=6 \mathrm{~m} \mathrm{~s}^{-1}$. Fig. (4) exemplifies the main corollaries of the turbulence effect on the ZW discussed in subsection $3.4-$ an increase of the wind velocity at high latitudes and its decrease at low latitudes including the formation of the negative wind velocity in the equatorial zone, as well as the shift of the location of the westerlies maximums to the poles if compared with the prediction of purely geostrophic approach.

\section{CONCLUSION}

A turbulence effect in the zonal winds (ZW) formation in the Earth's troposphere, foreseen by the RAT theory, is discussed. The analysis is performed within a model similar to the model explaining the turbulence effect in the formation of the Antarctic Circumpolar Current velocity field [11]. It has been shown that the accounted turbulence effect in- creases the wind velocity at higher latitudes, decreases the wind velocity at lower latitudes (if compared with the geostrophic ZW velocity prediction) and can explain easterlies in the equatorial zone. The increase of the wind velocity at higher latitudes and its decrease at lower latitudes is accompanied with a shift of the location of the westerlies maximums to the poles if compared with the geostrophic prediction. The suggested model does not require the geopotential local minimum at the Equator to explain the easterlies in the equatorial zone.

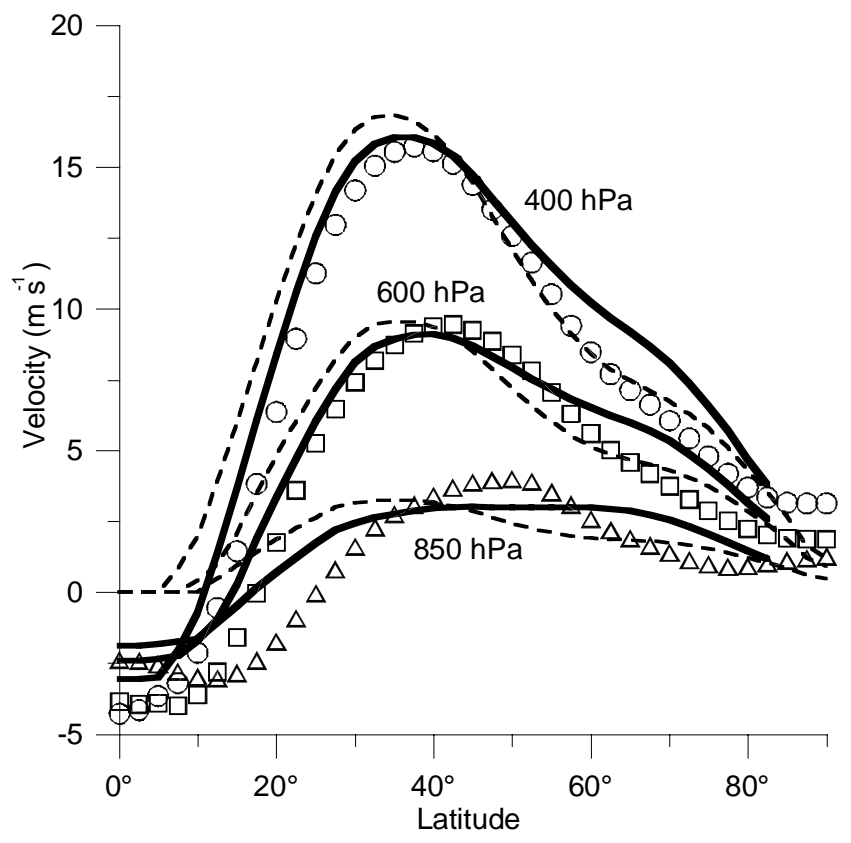

Fig. (4). Geostrophic $u^{g}(\vartheta)$ (dashed curves) and total $u$ (continuous curves) zonal velocities with observed zonal velocity data for 400 (circles), 600 (squares) and 850 (triangles) hPa from the data set compiled by Oort (1983).

\section{ACKNOWLEDGEMENTS}

Authors are grateful to Prof. Sirje Keevallik and Prof. Ain Kallis for their helpful comments.

\section{REFERENCES}

[1] Frederiksen JS. Nonlinear studies on the effects of topography on baroclinic zonal flows. Geophys Astrophys Fluid Dyn 1991; 59 (1-4): 57 - 82, doi: 10.1080/03091929108227773.

[2] Huss A. Numerical integration of a quasi-geostrophic atmospheric model with an asymmetric zonal current. Pure Appl Geophys 1966; 64 (1): 156-68, doi: 10.1007/BF00875541.

[3] Ellis RS, Turkington B. Analysis of statistical equilibrium models of geostrophic turbulence. J Appl Stochastic Anal 2002; 15 (4): 327-47.

[4] Shukla PK, Stenflo L. Generation of zonal flows by Rossby waves. Phys Lett A 2003; 307 (2-3): 85-177.

[5] Heinloo J. Phenomenological mechanics of turbulence. Valgus, Tallinn 1984; p. 245 (in Russian).

[6] Heinloo J. Turbulence mechanics. Introduction into general theory of turbulence. Estonian Academy of Science, Tallinn 1999 (in Russian).

[7] Heinloo J. Formulation of turbulence mechanics. Phys Rev E 2004; 69, 056317, doi: 10.1103/ Phys Rev E. 69056317.

[8] Heinloo, J. Setup of turbulence mechanics accounting for a preferred orientation of eddy rotation. Concept Phys 2008; 5 (2): 205-20. 
[9] Heinloo J. The description of externally influenced turbulence accounting for a preferred orientation of eddy rotation. Eur Phys $\mathbf{J}$ B 2008; 62: 471-76, doi: 10.1140/epjb/e2008-00187-8.

[10] Heinloo J, Toompuu A. Antarctic Circumpolar Currant as a density-driven flow. Proc Est Acad Sci Phys Math 2004; 53: 25265.

[11] Heinloo J, Toompuu A. Modeling a turbulence effect in formation of the Antarctic Circumpolar Current. Ann Geophys 2006; 24: 3191-96.

[12] Heinloo J, Võsumaa Ü. Rotationally anisotropic turbulence in the sea. Ann Geophys 1992; 10: 708-15.

[13] Võsumaa Ü, Heinloo J. Evolution model of the vertical structure of the active layer of the sea. J Geophys Res 1996; 101: 25635-46.

[14] Toompuu A, Heinloo J, Soomere T. Modelling the Gibraltar Salinity Anomaly. Oceanology 1989, English ed, Published by AGU in June 1990; 29: 698-702.

[15] Heinloo J. Eddy-driven flows over varying bottom topography. Proc Est Acad Sci Phys Math 2006; 55: 235-45.

[16] Heinloo J, Toompuu A. Eddy-to-mean energy transfer in geophysical jet flows. Proc Est Acad Sci Phys Math 2007; 56: 28394.

[17] Dahler JS. Transport phenomena in a fluid composed of diatomic moleculs. J Chem Phys 1959; 90: 1447-75.
[18] Dahler JS, Scriven LF. Angular momentum of continua. Nature 1961; 192: 36-7.

[19] Eringen AC. In: Kröner E, Ed. Mechanics of micromorphic continua: Mechanics of Generalized Continua, Springer-Verlag: Berlin 1968; pp. 18-33.

[20] Ariman T, Turk MA, Silvester DO. Microcontinuum fluid mechanics - review. Int J Eng Sci 1973; 11: 905-30.

[21] Richardson LF. Weather prediction by numerical process. Cambridge Univ Press: Cambridge 1922.

[22] Kolmogorov AN. The local structure of turbulence in incompressible viscous fluids for very large Reynolds numbers. C R Acad Sci URSS 1941; 30: 376-87.

[23] Holton JR. Dynamic meteorology. Academic Press 1992; p. 511.

[24] Oort AH. Global Atmospheric Circulation Statistics, 1958-1973, Rockville, MD, NOAA Professional Paper 14, 1983; p. 180.

[25] Fleming EL, Chandra S, Barnett JJ, Corney M. Zonal mean temperature, pressure, zonal wind and geopotential height as function of latitude, Adv Space Res 1990; 10: 11-59.

[26] Oort AH. Adequacy of the rawinsonde network for global circulation studies tested through numerical model output. Mon Weather Rev 1978; 106: 174-95.

(C) Heinloo and Toompuu; Licensee Bentham Open.

This is an open access article licensed under the terms of the Creative Commons Attribution Non-Commercial License (http://creativecommons.org/licenses/by$\mathrm{nc} / 3.0 /$ ) which permits unrestricted, non-commercial use, distribution and reproduction in any medium, provided the work is properly cited. 